\title{
One And The Same Route: Two Outstanding Electrodynamics
}

\author{
Antonio Accioly ${ }^{(1) *}$ and Hatsumi Mukai ${ }^{(2)}$ \\ (1) Instituto de Física Teórica, \\ Universidade Estadual Paulista, \\ Rua Pamplona,145, 01405-900 São Paulo, SP, Brazil \\ (2) Departamento de Física, \\ Fundação Universidade Estadual de Maringá, \\ Av. Colombo 5790, 87020-900 Maringá, Pr, Brazil
}

Received August 27, 1997

\begin{abstract}
We show that the same route that leads to Maxwell's electrodynamics leads also to Podolsky's electrodynamics, provided we start from Podolsky's electrostatic force law instead of the usual Coulomb's law.
\end{abstract}

\section{Introduction}

On the second edition of Jackson's seminal book on classical electrodynamics ${ }^{[1]}$, there is a section named "On the Question of Obtaining the Magnetic Field, Magnetic Force, and the Maxwell Equations from Coulomb's Law and Special Relativity", where it is shown in detail that any attempt to derive Maxwell equations from Coulomb's law of electrostatics and the laws of special relativity ends in failure unless one makes use of additional assumptions. What hypotheses are these? In an ingenious paper, Kobe ${ }^{[2]}$ gave the answer: all one needs to arrive at Maxwell equations is

(i) Coulomb's law;

(ii) the principle of superposition;

(iii) the assumption that electric charge is a conserved scalar (which amounts to assuming the independence of the observed charge of a particle on its speed $\left.^{[3]}\right)$;

(iv) the requirement of form invariance of the electrostatic field equations under Lorentz transformations, i.e. the electrostatic field equations are thought as covariant space-space components of covariant field equations.
Soon after Kobe's paper, Neuenschwander and Turner $^{[4]}$ obtained Maxwell equations by generalizing the laws of magnetostatics, which follow from the BiotSavart law and magnetostatics, to be consistent with special relativity.

The preceding considerations leads us to the interesting question: what would happen if we followed the same route as Kobe did, using an electrostatic force law other than the usual Coulomb's one? We shall show that if we start from the force law proposed by Podolsky ${ }^{[5]}$, i.e.,

$$
\mathbf{F}(\mathbf{r})=\frac{Q Q^{\prime}}{4 \pi}\left[\frac{1-e^{-r / a}}{r^{2}}-\frac{e^{-r / a}}{r a}\right] \frac{\mathbf{r}}{r}
$$

where $a$ is a positive parameter with dimension of length, $Q$ and $Q^{\prime}$ are the charges at $\mathbf{r}$ and $\mathbf{r}=\mathbf{0}$, respectively, and $\mathbf{F}(\mathbf{r})$ is the force on the particle with charge $Q$ due to the particle with charge $Q^{\prime}$ - and if we follow the steps previously outlined, we arrive at the outstanding electrodynamics derived by Podolsky ${ }^{[5]}$ in the early $40 s$. In other words, we shall show that the same route that leads to Maxwell equations leads also to Podolsky equations. A notable feature of Podolsky's

*e-mail: accioly@axp.ift.unesp.br 
generalized electrodynamics is that it is free of those infinities which are usually associated with a point source. For instance, (1) approaches a finite value $\frac{Q Q^{\prime}}{8 \pi a^{2}}$ as $r$ approaches zero. Thus, unlike Coulomb's law, Podolsky's electrostatic force law is finite in the whole space.

In Sec. II we obtain the equations that make up Podolsky's electrostatics. In Sec. III we arrive at Podolsky's field equations by generalizing the equations of Sec. II, so that they are form invariant under Lorentz transformations. For consistency, we show in Sec. IV that (1) is indeed the electrostatic force law related to Podolsky's theory. The conclusions are presented in Sec. V.

Natural units ( $\hbar=c=1)$ are used throughout. As far as the electromagnetic theories are concerned, we will use the Heaviside-Lorentz units with $c=1$.

\section{Podolsky's electrostatics}

As is well-known, the force on a text charge is proportional to its charge, all other properties of the force being assigned to the electric field $\mathbf{E}(\mathbf{r})$, which is defined by

$$
\mathbf{F}=Q \mathbf{E}(\mathbf{r})
$$

where $\mathbf{F}$ is force on the charge $Q$ situated at $\mathbf{r}$ and $\mathbf{E}(\mathbf{r})$ is the electric field at the position of this charge due to all other charges. (The source charge's coordinates will be distinguished from those of the field point, by a prime.) Accordingly, the electric field due to a point charge $Q^{\prime}$ situated at $\mathbf{r}^{\prime}$ is given by

$$
\mathbf{E}(\mathbf{r})=\frac{Q^{\prime}}{4 \pi}\left[\frac{1-e^{-R / a}}{R^{2}}-\frac{e^{-R / a}}{R a}\right] \frac{\mathbf{R}}{R},
$$

where $\mathbf{R}=\mathbf{r}-\mathbf{r}^{\prime}$. Note that this field is finite in the whole space. By the principle of superposition the electric field produced by the charge distribution $\rho\left(\mathbf{r}^{\prime}\right)$ is

$$
\mathbf{E}(\mathbf{r})=\int d^{3} \mathbf{r}^{\prime} \frac{\rho\left(\mathbf{r}^{\prime}\right)}{4 \pi}\left[\frac{1-e^{-R / a}}{R^{2}}-\frac{e^{-R / a}}{R a}\right] \frac{\mathbf{R}}{R},
$$

where the integration is carried out over all space. ( $\rho$ must of course vanish at sufficient large $\mathbf{r}$, making the volume of integration less than infinity.)

The preceding expression for the electric field arising from a charge distribution may be easily expressed as a gradient of a scalar integral as follows

$$
\mathbf{E}(\mathbf{r})=-\int d^{3} \mathbf{r}^{\prime} \frac{\rho\left(\mathbf{r}^{\prime}\right)}{4 \pi} \nabla\left[\frac{1-e^{-R / a}}{R}\right] .
$$

Since $\nabla$ acts only on the unprimed coordinates, we may take it outside the integral (4) to obtain

$$
\mathbf{E}(\mathbf{r})=-\nabla\left[\int d^{3} \mathbf{r}^{\prime} \frac{\rho\left(\mathbf{r}^{\prime}\right)}{4 \pi} \frac{1-e^{-R / a}}{R}\right] .
$$

Taking into account that the curl of any gradient vanishes, we have immediately

$$
\nabla \times \mathbf{E}=0
$$

which shows that Podolsky's electrostatic field, like Maxwell's one, is conservative. If the divergence of (3) is taken, the result is

$$
\begin{aligned}
\boldsymbol{\nabla} \cdot \mathbf{E}(\mathbf{r}) & =\int d^{3} \mathbf{r}^{\prime} \frac{\rho\left(\mathbf{r}^{\prime}\right)}{4 \pi} \nabla \cdot\left[\left(\frac{1-e^{-R / a}}{R^{2}}-\frac{e^{-R / a}}{R a}\right) \frac{\mathbf{R}}{R}\right] \\
& =\int d^{3} \mathbf{r}^{\prime} \frac{\rho\left(\mathbf{r}^{\prime}\right)}{4 \pi} \frac{e^{-R / a}}{R a^{2}},
\end{aligned}
$$

where use has been made of the identity

$$
\nabla \cdot(\Psi \mathbf{A})=\nabla \Psi \cdot \mathbf{A}+\Psi \nabla \cdot \mathbf{A} .
$$

Now, taking the Laplacian of (7), and using the identity (see appendix)

$$
\nabla^{2} \frac{e^{-r / a}}{R}=\frac{e^{-r / a}}{R a^{2}}-4 \pi \delta^{3}(\mathbf{R})
$$


yields

$$
\begin{aligned}
\nabla^{2}[\boldsymbol{\nabla} \cdot \mathbf{E}(\mathbf{r})] & =\int d^{3} \mathbf{r}^{\prime} \frac{\rho\left(\mathbf{r}^{\prime}\right)}{4 \pi a^{2}} \nabla^{2}\left(\frac{e^{-R / a}}{R}\right) \\
& =\frac{1}{a^{2}} \int d^{3} \mathbf{r}^{\prime} \frac{\rho\left(\mathbf{r}^{\prime}\right)}{4 \pi} \frac{e^{-R / a}}{R a^{2}}-\int d^{3} \mathbf{r}^{\prime} \frac{\rho\left(\mathbf{r}^{\prime}\right)}{a^{2}} \delta^{3}\left(\mathbf{r}-\mathbf{r}^{\prime}\right) \\
& =\frac{1}{a^{2}}[\nabla \cdot \mathbf{E}(\mathbf{r})-\rho(\mathbf{r})] .
\end{aligned}
$$

So,

$$
\left[1-a^{2} \nabla^{2}\right] \nabla \cdot \mathbf{E}(\mathbf{r})=\rho(\mathbf{r})
$$

Equations (6) and (8) are the fundamental laws of Podolsky's electrostatics. We will digress slightly at this stage to analyze an interesting feature of Podolsky's electrostatics. In this vein, we compute the flux of the electrostatic field across a spherical surface of radius $R$ with a charge $Q$ at its center. Using (2) we arrive at the result

$$
\oint \mathbf{E} \cdot d \mathbf{S}=Q\left[1-\left(1+\frac{R}{a}\right) e^{-R / a}\right]
$$

which tells us that

$$
\oint \mathbf{E} \cdot d \mathbf{S}= \begin{cases}0, & R \ll a \\ Q, & R \gg a\end{cases}
$$

Therefore, a sphere of radius $R \ll a$, unlike what happens in Maxwell's theory, shields its exterior from the field due to a charge placed at its center. We remark that in Maxwell's electrostatics a closed hollow conductor shields its interior from fields due to charges outside, but does not shield its interior from the field due to charges placed inside $i^{[6]}{ }^{[6]}$ Note, however, that in order not to conflict with well established results of quantum electrodynamics, the parameter a must be small. Incidentally, it was shown recently that this parameter is of the order of magnitude of the Compton wavelength of the neutral vector boson $z, \lambda(z) \approx 2.16 \times 10^{-16} \mathrm{~cm}$, which mediates the unified and electromagnetic interactions ${ }^{[7]}$. After this parenthesis, let us return to our main subject. Equations (6) and (8) are now ready to be generalized using special relativity and the hypotheses that electric charge is a conserved scalar. We shall do that in the next section.

\section{Podolsky's field equations}

To begin with let us establish some conventions and notations to be used from now on. We use the metric tensor

$$
\eta^{\mu \nu}=\eta_{\mu \nu}=\left(\begin{array}{cccc}
1 & 0 & 0 & 0 \\
0 & -1 & 0 & 0 \\
0 & 0 & -1 & 0 \\
0 & 0 & 0 & -1
\end{array}\right)
$$

with Greek indices running over 0,1,2,3. Roman indices - $i, j, e t c$, - denote only the three spatial components. Repeated indices are summed in all cases. The space-time four vectors (contravariant vectors) are $x^{\mu}=(t, \mathbf{x})$, and the covariant vectors, as a consequences are $x_{\mu}=(t,-\mathbf{x})$. The four-velocities are found, according to

$$
\begin{aligned}
u^{\mu} & =\frac{d x^{\mu}}{d \tau} \\
& =\gamma(1, \mathbf{v}) \\
u_{\mu} & =\gamma(1,-\mathbf{v})
\end{aligned}
$$

where $\tau$ is the proper time $\left(d \tau^{2}=d t^{2}-d \mathbf{x}^{2}\right)$, and $\gamma$ denotes $d t / d \tau=\left(1-\mathbf{v}^{2}\right)^{-1 / 2}$.

Let us then generalize (6) so that it satisfies the requirement of form invariance under Lorentz transformations. To do that, we write the mentioned equation in terms of the Levi-Civita density $\varepsilon^{n m l}$, which equals $+1(-1)$ if $n, m, l$ is an even(odd) permutation of $1,2,3$, and vanishes if two indices are equal. The curl equation becomes

$$
\varepsilon^{j k l} \partial_{k} E_{l}=0
$$


It we define the quantities

$$
F^{0 i}=-F_{0 i}=E^{i}=-E_{i},
$$

(10) can be rewritten as

$$
\varepsilon^{j k l} \partial_{k} F_{0 l}=0
$$

We imagine now the curl law to be the space-space components of a manifestly covariant field equation (invariance under Lorentz transformations). As a result, we get

$$
\varepsilon^{\mu \nu \alpha \beta} \partial_{\nu} F_{\alpha \beta}=0
$$

where $\varepsilon^{\mu \nu \alpha \beta}$ is a completely antisymmetric tensor of rank four with $\varepsilon^{0123}=+1$.

Of course, this generalization introduces the components $F_{00}, F_{l 0}$, and $F_{l k}$, for which at this point we lack a physical interpretation. Note that the $F_{0 i}$ are not necessarily static anymore.

On the other hand, as is well-known, the charge density $\rho$ is defined as the charge per unit of volume, which has as a consequence that the charge $d q$ in an element of volume $d^{3} \mathbf{x}$ is $d q=\rho d^{3} \mathbf{x}$. Since $d q$ is a Lorentz scalar ${ }^{[3]}$, $\rho$ transforms as the time-component of a four-vector, namely, the time-component of the charge-current fourvector $j^{\mu}=(\rho, \mathbf{j})$. The electric charge, in turn, is conserved locally ${ }^{[3]}$, which implies that it obeys a continuity equation

$$
\partial_{\mu} j^{\mu}=0
$$

(8) can now be rewritten as

$$
\left[1+a^{2} \partial_{i} \partial^{i}\right] \partial_{j} E^{j}=j^{0},
$$

where $\partial_{i} \equiv \partial / \partial x^{i}$ and $\partial^{i} \equiv \partial / \partial x_{i}$. Note that $\partial_{i}=-\partial^{i}$. Using (11), yields

$$
\left[1+a^{2} \partial_{i} \partial^{i}\right] \partial_{j} F^{0 j}=j^{0}
$$

In order that the left-hand side of the preceding equation transforms as the time-component of a four-vector, we must write it as

$$
\left[1+a^{2} \square\right] \partial_{\mu} F^{0 \mu}=j^{0}
$$

where

$$
\begin{aligned}
\square & =\partial_{\mu} \partial^{\mu} \\
& =\eta^{\mu \nu} \partial_{\mu} \partial_{\nu} \\
& =\partial^{2} / \partial t^{2}-\nabla^{2}
\end{aligned}
$$

The requirement of form invariance of this equation under Lorentz transformations leads then to the following result

$$
\left[1+a^{2} \square\right] \partial_{\nu} F^{\mu \nu}=j^{\mu}
$$

Imagine now a particle of mass $m$ and charge $Q$ at rest in a lab frame where there is an electrostatic field $\mathbf{E}$. Newton's second law allows us to write

$$
\frac{d \mathbf{p}}{d t}=Q \mathbf{E}
$$

In terms of the proper time this becomes

$$
\begin{aligned}
\frac{d \mathbf{p}}{d \tau} & =Q \gamma \mathbf{E} \\
& =Q u^{0} \mathbf{E}
\end{aligned}
$$

where $u^{0}$ is the time part of the velocity four-vector $u^{\mu}$. For the component along de $x^{i}$ direction, we have

$$
\frac{d p^{i}}{d \tau}=Q u_{0} F^{0 i}
$$

In order that the right-hand side of this equation transforms like a space-component of a four-vector, it must be rewritten as

$$
\frac{d p^{i}}{d \tau}=Q u_{\nu} F^{\nu i}
$$

whose covariant generalization is

$$
\frac{d p^{\mu}}{d \tau}=Q u_{\nu} F^{\nu \mu}
$$

If (16) is multiplied by $p_{\mu}=m u_{\mu}$, where $m$ is the rest mass, the result is

$$
\frac{1}{2} \frac{d}{d \tau}\left(p_{\mu} p^{\mu}\right)=Q m u_{\mu} u_{\nu} F^{\nu \mu}
$$

However,

$$
\begin{aligned}
p_{\mu} p^{\mu} & =m^{2} \gamma^{2}\left(1-\mathbf{v}^{2}\right) \\
& =m^{2} \gamma^{2} / \gamma^{2} \\
& =m^{2}
\end{aligned}
$$


Therefore, we come to the conclusion that

$$
u_{\mu} u_{\nu} F^{\nu \mu}=0
$$

Using this result Kobe ${ }^{[2]}$ and Neuenschwander and Turner ${ }^{[3]}$ showed that $F^{\mu \nu}$ is an antisymmetric tensor $\left(F^{\mu \nu}=-F^{\nu \mu}\right)$. Since $F^{\mu \nu}$ is an antisymmetric tensor of second rank, it has only six independent components, three of which have already been specified. We name therefore the remaining components

$$
B^{i}=\frac{1}{2} \epsilon^{i l m} F_{l m}
$$

Note that $F^{k l}=-\epsilon^{k l j} B_{j}$. Writing out the components of (17) explicitly,

$$
\begin{aligned}
& B^{1}=F_{23}=F^{23}=-B_{1}, \\
& B^{2}=-F_{13}=-F^{13}=-B_{2} \\
& B^{3}=F_{12}=F^{12}=-B_{3} .
\end{aligned}
$$

Hence, a clever physicist who were only familiar with Podolsky's electrostatics and special relativity could predict the existence of the magnetic field $\mathbf{B}$, which naturally still lacks physical interpretation.

The content of (12) and (14) can now be seen. For $\mu=0$, (12) gives

$$
\boldsymbol{\nabla} \cdot \mathbf{B}=0
$$

showing that there are no magnetic monopoles in Podolsky's electrodynamics, while for $\mu=i$ we obtain

$$
\boldsymbol{\nabla} \times \mathbf{E}=-\frac{\partial \mathbf{B}}{\partial t}
$$

which says that time-varying magnetic fields can be produced be $\mathbf{E}$ fields with circulation.

The components $\mu=0$ and $\mu=i$ of (14) give, respectively,

$$
\begin{array}{r}
\left(1+a^{2} \square\right) \boldsymbol{\nabla} \cdot \mathbf{E}=\rho, \\
\left(1+a^{2} \square\right)\left[\boldsymbol{\nabla} \times \mathbf{B}-\frac{\partial \mathbf{E}}{\partial t}\right]=\mathbf{j} ;
\end{array}
$$

which are nothing but a generalization of Gauss and Ampère- Maxwell laws in this order.

For $\nu=i,(16)$ becomes

$$
\frac{d \mathbf{p}}{d t}=Q(\mathbf{E}+\mathbf{v} \times \mathbf{B})
$$

containing the Lorentz force. For $\nu=0,(15)$ assumes the form

$$
\frac{d U}{d t}=Q \mathbf{v} \cdot \mathbf{E}
$$

where $U=p^{0}$ is the particle's energy. Accordingly, our smart physicist, who was able to predict the $\mathbf{B}$ field only from its knowledge of electrostatics and special relativity, can now-by making judicious use of (22) and (23)observe, measure and distinguish the $\mathbf{B}$ field from the $\mathbf{E}$ field of (15). The new field couples to moving electric charge, does not act on a static charged particle, and, unlike the electrostatic field, is capable only of changing the particle's momentum direction.

Equations (18-21) make up Podolsky's higher-order field equations. Of course, in the limit $a=0$, all the preceding arguments apply equally well to Maxwell's theory.

Two comments fit in here:

(1) equation (14) is consistent with the continuity equation (13). In fact, if the divergence of (14) is taken, we obtain

$$
\left[1+a^{2} \square\right] \partial_{\mu} \partial_{\nu} F^{\mu \nu}=\partial_{\mu} j^{\mu}
$$

Since $F^{\mu \nu}$ is an antisymmetric tensor $\partial_{\mu} \partial_{\nu} F^{\mu \nu}$ is identically zero. On the other hand, according to (14), $\partial_{\mu} j^{\mu}=0$. Thus, the equation in hand is identically zero;

(2) as was recently shown ${ }^{[8]}$, it is not necessary to introduce a formula for the force density $f^{\mu}$ representing the action of the field on a text particle. We have only to assume that $\left(-f^{\mu}\right)$ is the simplest contravariant vector constructed with the current $j^{\mu}$ and a suitable derivative of the field $F^{\mu \nu}$. Applying this simplicity criterion to Podolsky's electrodynamics, we promptly obtain

$$
f^{\mu}=-F^{\mu \nu} j_{\nu}
$$

where, as we have already mentioned, $j^{\mu}=(\rho, \mathbf{j})$. Therefore,

$$
f^{0}=-F^{0 i} j_{i}=\mathbf{E} \cdot \mathbf{j}
$$


and

$$
\begin{array}{r}
f^{k}=-F^{k \beta} j_{\beta}=F^{0 k} j_{0}+F^{i k} j_{i} \\
=F^{0 k} j_{0}+\epsilon^{k i l} j_{i} B_{l}=(\rho \mathbf{E}+\mathbf{j} \times \mathbf{B})^{k}
\end{array}
$$

Thus, the force density for Podolsky's electrodynamics is the same as that for Maxwell's electrodynamics, namely, the well-known Lorentz force density.

\section{Finding the force law for Podolsky's electrostatics}

We show now that (1) is indeed the force law for Podolsky's electrostatics. ¿From (5) it follows that

$$
\mathbf{E}=-\nabla V
$$

where

$$
V(\mathbf{r}) \equiv \int d^{3} \mathbf{r}^{\prime} \frac{\rho\left(\mathbf{r}^{\prime}\right)}{4 \pi} \frac{1-e^{-R / a}}{R} .
$$

Eq. (8) can then be rewritten as

$$
\left(1-a^{2} \nabla^{2}\right) \nabla^{2} V(\mathbf{r})=-\rho(\mathbf{r})
$$

For a charge $Q$ at the origin of the radius vector this equation reduces to

$$
\left(1-a^{2} \nabla^{2}\right) \nabla^{2} V(\mathbf{r})=-Q \delta^{3}(\mathbf{r})
$$

We solve this equation using the Fourier transform method. First we define $\tilde{V}(\mathbf{k})$ as follows:

$$
\begin{aligned}
V(\mathbf{r}) & =\frac{1}{(2 \pi)^{3 / 2}} \int d^{3} \mathbf{k} e^{-i \mathbf{k} \cdot \mathbf{r}} \tilde{V}(\mathbf{k}), \\
\tilde{V}(\mathbf{k}) & =\frac{1}{(2 \pi)^{3 / 2}} \int d^{3} \mathbf{r} e^{i \mathbf{k} \cdot \mathbf{r}} V(\mathbf{r})
\end{aligned}
$$

where $d^{3} \mathbf{k}$ and $d^{3} \mathbf{r}$, respectively, stands for volumes in the three-dimensional $k$-space and the coordinate space. If we substitute (26) into (25) and take into account that

$$
\delta^{3}(\mathbf{r})=\frac{1}{(2 \pi)^{3}} \int d^{3} \mathbf{k} e^{-i \mathbf{k} \cdot \mathbf{r}}
$$

we promptly obtain

$$
\tilde{V}(\mathbf{k})=\frac{Q}{(2 \pi)^{3 / 2}} \frac{M^{2}}{k^{2}\left(k^{2}+M^{2}\right)}
$$

where $M^{2} \equiv 1 / a^{2}$. So,

$$
V(\mathbf{r})=\frac{Q M^{2}}{(2 \pi)^{3}} \int d^{3} \mathbf{k} \frac{e^{-i \mathbf{k} \cdot \mathbf{r}}}{k^{2}\left(k^{2}+M^{2}\right)}
$$

Since the orientation of our coordinate system is arbitrary, we may choose the $\mathbf{z}$-axis along $\mathbf{r}$ and obtain

$$
V(\mathbf{r})=\frac{Q M^{2}}{(2 \pi)^{3}} \int_{0}^{\infty} k^{2} d k \int_{-1}^{+1} \frac{e^{-i k r \cos \theta}}{\left(k^{2}+M^{2}\right) k^{2}} d(\cos \theta) \int_{0}^{2 \pi} d \phi
$$

where $r=|\mathbf{r}|, k=|\mathbf{k}|$, and $(\theta, \phi)$ are conventional spherical polar coordinates. As a consequence,

$$
\begin{aligned}
V(\mathbf{r} & =\frac{Q M^{2}}{(2 \pi)^{2}} \int_{-\infty}^{+\infty} \frac{\sin k r}{k r} \frac{d k}{k^{2}+M^{2}} \\
& =\frac{Q M^{2} r}{(2 \pi)^{2}} \int_{-\infty}^{\infty} \frac{\sin x}{x} \frac{d x}{x^{2}+M^{2} r^{2}}
\end{aligned}
$$

But,

$$
\frac{1}{x} \frac{1}{x^{2}+M^{2} r^{2}}=\frac{1}{M^{2} r^{2}}\left(\frac{1}{x}-\frac{x}{x^{2}+M^{2} r^{2}}\right) .
$$

Therefore,

$$
V(\mathbf{r})=\frac{Q}{(2 \pi)^{2}} r\left[I_{1}-I_{2}\right]
$$

Integral (28) may be found in any textbook on the theory of functions of a complex variable ${ }^{[9]}$. It can also be 
carried out by means of a trivial trick ${ }^{[10]}$. Indeed, let

$$
\begin{aligned}
I(\alpha) & =\int_{0}^{\infty} \frac{e^{-\alpha x} \sin x}{x} d x \text { so that } I_{1}=2 I(0) \\
\frac{d I(\alpha)}{d \alpha} & =-\int_{0}^{\infty} e^{-\alpha x} \sin x d x=\frac{-1}{1+\alpha^{2}} \\
I(\alpha) & =-\int \frac{d \alpha}{1+\alpha^{2}}=C-\tan ^{-1} \alpha .
\end{aligned}
$$

But $I(\infty)=0$. Therefore $C=\pi / 2 . I(\alpha)=\frac{\pi}{2}-\tan ^{-1} \alpha$ and $I_{1}=2 I(0)=\pi$.

Integral (29) can be easily evaluated by the method of contour integration ${ }^{[11]}$. Consider in this vein $\int_{\gamma} \frac{z e^{i z} d z}{z^{2}+M^{2} r^{2}}$, where the contour of integration $\gamma$ was chosen to be the real axis and a semicircle of infinite radius in the upper half plane. Along the real axis the integral is $I_{2}$. In the large semicircle in the upper half plane we get zero, since $\exp (i z) \rightarrow 0$ for $z \rightarrow i \infty$.

The residue of

$$
\frac{z e^{i z}}{z^{2}+M^{2} r^{2}}=\frac{z e^{i z}}{2 M r i}\left[\frac{1}{z-M r i}-\frac{1}{z+M r i}\right]
$$

at $z=M r i$ (which is the only pole that lies inside the contour of integration) is $\frac{e^{-M r}}{2}$. Hence,

$$
I_{2}=\operatorname{Im}\left(2 \pi i \frac{e^{-r M}}{2}\right)=\pi e^{-r M}
$$

As a result,

$$
\begin{aligned}
V(\mathbf{r}) & =\frac{Q}{(2 \pi)^{2} r}\left[\pi-\pi e^{-r M}\right] \\
& =\frac{Q}{4 \pi} \frac{1-e^{-r / a}}{r} .
\end{aligned}
$$

Accordingly, the electric field due to a charge $Q$ at the origin is given by

$$
\begin{aligned}
\mathbf{E}(\mathbf{r}) & =-\nabla V \\
& =-\nabla\left(\frac{Q}{4 \pi} \frac{1-e^{-r / a}}{r}\right) \\
& =\frac{Q}{4 \pi}\left[\frac{1-e^{-r / a}}{r^{2}}-\frac{e^{-r / a}}{r a}\right] \frac{\mathbf{r}}{r} .
\end{aligned}
$$

It follows then the force law for Podolsky's electrostatics is

$$
\mathbf{F}(\mathbf{r})=\frac{Q Q^{\prime}}{4 \pi}\left[\frac{1-e^{-r / a}}{r^{2}}-e^{-r / a} r a\right] \frac{\mathbf{r}}{r},
$$

which is nothing but the force law for which we were looking (see Eq. (1)).

Recently an algorithm was devised which allows one to obtain the energy and momentum related to a given field in a simple way ${ }^{[8]}$. Using this prescription we can show that in the framework of Podolsky's electrostatics the energy is given by

$$
\mathcal{E}_{\text {field }}=\frac{1}{2} \int d^{3} \mathbf{x}\left[\mathbf{E}^{2}+a^{2}(\boldsymbol{\nabla} \cdot \mathbf{E})^{2}\right] .
$$

Making use of the expression for the electrostatic field we have just found, we promptly obtain

$$
\varepsilon_{\text {field }}=Q^{2} / 2 a,
$$

which tells us that the energy for the field of a point charge has a finite value in the whole space. This is indeed a nice feature of Podolsky's generalized electrodynamics.

\section{Final remarks}

Despite the simplicity of its fundamental assumptions, Podolsky's model has been little noticed. Currently some of its aspects have been further studied in the literature ${ }^{[7,8,12,13]}$. In particular, the classical self-force acting on a point charge in Podolsky's model was evaluated and it was shown that in this model, unlike what happens in Maxwell's electrodynamics, the electromagnetic mass is finite and enters the particle's equation of motion in a form consistent with special relativity.

To conclude we call attention to the fact the same assumptions that lead to Maxwell's equations lead also to Podolsky's equations, provided we start from a generalization of the Coulomb's law instead of the usual Coulomb's law. Yet, in spite of the great similarity between the two theories, Podolsky's generalized electrodynamics leads to results that are free of those infinities which are usually associated with a point source. 


\section{Appendix: An important identity involving $\delta$ functions}

An useful identity involving $\delta$ functions is

$$
\nabla^{2} \frac{e^{-R / a}}{R}=\frac{e^{-R / a}}{a^{2} R}-4 \pi \delta^{3}\left(\mathbf{r}-\mathbf{r}^{\prime}\right)
$$

where $R=\left|\mathbf{r}-\mathbf{r}^{\prime}\right|$.

To prove this let us consider

$$
\int_{V} f\left(\mathbf{r}^{\prime}\right) \nabla^{2}\left(\frac{e^{-R / a}}{R}\right) d^{3} \mathbf{r}^{\prime}
$$

If $\mathbf{r}$ is not in the region $V$ over which we are integrating, it never coincides with $\mathbf{r}^{\prime}$, and it is easily verified by direct differentiation that $\nabla^{2} \frac{e^{-R / a}}{R}=\frac{e^{-R / a}}{a^{2} R}$. Any region $V$ that contains $\mathbf{r}^{\prime}$ may be subdivided into a small sphere of radius $\rho$ centered on $\mathbf{r}$ surrounded by surface $S$ and the remaining volume where $\nabla^{2} \frac{e^{-R / a}}{R}=\frac{e^{-R / a}}{a^{2} R}$. Hence,

$$
\begin{aligned}
\int_{V} f\left(\mathbf{r}^{\prime}\right) \nabla^{2}\left(\frac{e^{-R / a}}{R}\right) d^{3} \mathbf{r}^{\prime} & =\int_{\text {sphere }} f\left(\mathbf{r}^{\prime}\right) \nabla^{2}\left(\frac{e^{-R / a}}{R}\right) d^{3} \mathbf{r}^{\prime} \\
& +\int_{\substack{\text { remaining } \\
\text { volume }}} f\left(\mathbf{r}^{\prime}\right) \frac{e^{-R / a}}{a^{2} R} d^{3} \mathbf{r}^{\prime}
\end{aligned}
$$

Now, according to the mean value theorem,

$$
\int_{\text {sphere }} f\left(\mathbf{r}^{\prime}\right) \nabla^{2}\left(\frac{e^{-R / a}}{R}\right) d^{3} \mathbf{r}^{\prime}=f(\xi) \int_{\text {sphere }} \nabla^{2}\left(\frac{e^{-R / a}}{R}\right) d^{3} \mathbf{r}^{\prime},
$$

where $\xi$ is some point in the sphere. Applying the divergence theorem to the last integral, we obtain

$$
\int_{\text {sphere }} \nabla \cdot \nabla\left(\frac{e^{-R / a}}{R}\right) d^{3} \mathbf{r}^{\prime}=\int_{\mathrm{S}}\left(\frac{e^{-R / a}}{R^{2}}+\frac{e^{-R / a}}{R a}\right) \frac{\mathbf{R}}{R} \cdot d \mathbf{S}^{\prime} .
$$

Note that $\nabla_{\mathbf{r}}=-\nabla_{\mathbf{r}^{\prime}}$. On the other hand,

$$
\int_{\mathrm{S}}\left(\frac{e^{-R / a}}{R^{2}}+\frac{e^{-R / a}}{R a}\right) \frac{\mathbf{R}}{R} \cdot d \mathbf{S}^{\prime}=-4 \pi\left[e^{-R / a}+\frac{\rho e^{-\rho / a}}{a}\right] .
$$

As $\rho$ tends to $0, \xi$ must approach $\mathbf{r}$, so that

$$
\int_{\text {sphere }} f\left(\mathbf{r}^{\prime}\right) \nabla^{2}\left(\frac{e^{-R / a}}{R}\right) d^{3} \mathbf{r}^{\prime}=-4 \pi f(\mathbf{r}) .
$$

Thus,

$$
\begin{aligned}
\int d^{3} \mathbf{r}^{\prime} f\left(\mathbf{r}^{\prime}\right) \nabla^{2} \frac{e^{-R / a}}{R} & =-4 \pi f(\mathbf{r})+\int d^{3} \mathbf{r}^{\prime} \frac{e^{-R / a}}{a^{2} R} f\left(\mathbf{r}^{\prime}\right) \\
& =\int d^{3} \mathbf{r}^{\prime}\left[\frac{e^{-R / a}}{a^{2} R}-4 \pi \delta^{3}\left(\mathbf{r}-\mathbf{r}^{\prime}\right)\right] f\left(\mathbf{r}^{\prime}\right) .
\end{aligned}
$$




\section{References}

9. F. W. Byron, Jr, and R. W. Fuller, Mathematics of Classical and Quantum Physics (Addison-Wesley

1. J. D. Jackson, Classical Electrodynamics (Wiley, Publishing Company, New York, 1970), volume New York, 1975), 2nd. ed., pp. 578-581.

2. D. H. Kobe, Am. J. Phys 54, 631 (1986).

3. See Ref. 1, pp. 547-552.

4. D. E. Neuenschwander and B. N. Turner, Am. J. Phys. 60, 35 (1992). two, pp. 366-367.

10. Jon Mathews and R.L. Walker, Mathematical Methods of Physics (W.A. Benjamin, Inc., New York, 1965) p. 58.

5. B. Podolsky, Phys. Rev. 62, 66 (1942); B.Podolsky and P. Schwed, Rev. Mod. Phys, 20, 40 (1948).

6. See Ref. 1, p.49.

7. J. Frenkel and R. B. Santos, "The self-force on point charged particles in Podolsky's generalized electrodynamics," (to be published).

11. See Ref. 9, pp. 362-365.

12. C. A. P. Galvão and B. M. Pimentel, Can. J. Phys. 66, 460 (1988); J. Barcelos-Neto, C. A. P. Galvão, C. P. Natividade, Z. Phys. C52, 559 (1991); L. V. Belvedere, C. P. Natividade, C. A. P. Galvão, Z. Phys. C56, 609 (1992).

13. A. J. Accioly and H. Mukai, Z.Phys. C 75, 187 (1997).

8. Antonio Accioly, Am. J. Phys. 65, 882 (1997). 